\title{
Die Handelsbeziehungen der Schweiz zu den ostasiatischen Entwicklungsländern
}

Rudolf Ramsauer

\section{(2) OpenEdition}

12 Journals

Electronic version

URL: http://journals.openedition.org/sjep/1089

ISSN: 1663-9677

Publisher

Institut de hautes études internationales et du développement

Printed version

Date of publication: 1 décembre 1989

Number of pages: 137-155

ISSN: 1660-5926

\section{Electronic reference}

Rudolf Ramsauer, «Die Handelsbeziehungen der Schweiz zu den ostasiatischen

Entwicklungsländern », Schweizerisches Jahrbuch für Entwicklungspolitik [Online], 8 | 1989, Online erschienen am: 13 März 2013, abgerufen am 07 September 2020. URL : http:// journals.openedition.org/sjep/1089 


\title{
Die Handelsbeziehungen der Schweiz zu den ostasiatischen Entwicklungsländern
}

\author{
Rudolf Ramsauer
}

Eine Beschreibung der schweizerischen Handelsbeziehungen zu den ostasiatischen Entwicklungsländern wirtt sogleich definitorische Fragen auf, welche die Problematik und das besondere Interesse des Themas ins Licht stellen, gleichzeitig aber auch Zweifel darüber aufkommen lassen, ob hier überhaupt ein einheitlicher Gegenstand abgehandelt wird. Einige klärende Vorbemerkungen dürften deshalb angebracht sein.

\section{Vorbemerkungen}

Erstens, dieser Beitrag orientiert sich nicht an der genauen Abgrenzung eines geographischen Raumes, der sich mit "Ostasien" bezeichnen lässt. Er geht von der einfachen Feststellung aus, dass die wirtschaftliche Entwicklung im Fernen Osten gesamthaft eine Dynamik erfährt und vorlebt, die einzigartig ist und deren Fortgang kaum bezweifelt wird. Ostasiens gesamter wirtschaftlicher "Output" entsprach 1960 rund der Hälfte der europäischen oder einem Drittel der nordamerikanischen Produktion; bis zum Ende dieses Jahrhunderts könnte Ostasien mit Westeuropa und Nordamerika gleichgezogen haben. Eine gigantische globale Verschiebung von Reichtum und wirtschaftlichen Ressourcen ist gegenwärtig im Gange, wie die "Financial Times" kürzlich kommentiert hat. Solche Aussagen sind zwar sehr summarisch, deuten aber auf weltwirtschaftliche Entwicklungen hin, welche auf die Struktur des internationalen Handels und die Zukunft der internationalen Handelspolitik grossen Einfluss haben werden.

Zweitens, die Vision eines "pazifischen 21. Jahrhunderts" - nach dem "atlantischen 20. Jahrhundert" -, welche der einen Phantasie beflügelt und der an- 
dern Ängste wachruft, mag mehr oder weniger ernst genommen werden. Auf jeden Fall werden im pazifischen Raum sehr ernsthaft Kooperationsmodelle diskutiert, die eines Tages konkrete wirtschafts- und handelspolitische Konsequenzen haben könnten. Es sei hier lediglich an die Initiative des früheren japanischen Premierministers Nakasone erinnert, ein «Pacific Forum for Economic and Cultural Cooperation" zu gründen, welches eine engere und operationellere Form der Zusammenarbeit ermöglichen sollte als die seit 1980 bestehende private "Pacific Economic Cooperation Conference" (1). Dieser Gegenstand kann hier nicht näher abgehandelt werden, seine Bedeutung für die schweizerische Handelspolitik ist aber unbestreitbar und zudem von hoher Aktualität.

Drittens, von ostasiatischen "Entwicklungsländern" zu sprechen, fällt schwer, welche Entwicklungskriterien auch immer gewählt werden. Orientiert man sich an der klassischen Grösse des Bruttosozialprodukts pro Kopf der Bevölkerung, wird man etwa Burma mit seinen 190 US\$ (1985) zu den allerärmsten Entwicklungsländern zählen müssen, während Singapur mit 7420 US\$ durchaus der Kategorie der mittelreichen Industrieländer zuzuordnen ist. Portugal, Griechenland, Spanien, Irland, Italien und Neuseeland haben 1985 allesamt niedrigere Pro-Kopf-Einkommen erwirtschaftet als Singapur. Die nachfolgende Tabelle gibt die entsprechenden Kennziffern für die uns hier interessierenden Länder (2) wieder:

\begin{tabular}{|lcc|c|}
\hline \multicolumn{3}{|c|}{$\begin{array}{l}\text { Bruttosozialprodukt pro Kopf } \\
\text { der Bevölkerung (3) }\end{array}$} & $\begin{array}{l}\text { Bevölkerung, } \\
\text { 1985, in Mio (3) }\end{array}$ \\
\hline & $\begin{array}{c}\text { 1985, } \\
\text { in }\end{array}$ & $\begin{array}{c}\text { jährliche durch- } \\
\text { schnittliche } \\
\text { Wachstumsrate, } \\
\text { 1965-1985 }\end{array}$ & \\
Singapur & S & $7,6 \%$ & \\
Hongkong & 7420 & $6,1 \%$ & 2,6 \\
Südkorea & 2150 & $6,6 \%$ & 5,4 \\
Malaysia & 2000 & $4,4 \%$ & 41,1 \\
Thailand & 800 & $4,0 \%$ & 15,6 \\
Philippinen & 580 & $2,3 \%$ & 51,7 \\
Indonesien & 530 & $4,8 \%$ & 54,7 \\
China & 310 & $4,8 \%$ & 162,2 \\
Indien & 270 & $1,7 \%$ & 1040,3 \\
Burma & 190 & $2,4 \%$ & 765,1 \\
Bangladesh & 150 & $0,4 \%$ & 36,9 \\
Dazu als Vergleich: & & & 100,6 \\
Japan & 11300 & $4,7 \%$ & \\
USA & 16690 & $1,7 \%$ & 120,8 \\
Schweiz & 16370 & $1,4 \%$ & 239,3 \\
\hline
\end{tabular}


Diese beiden Zahlenreihen haben die aufschlussreiche Eigenschaft, dass sie beide abnehmende Tendenz zeigen; d.h. die heute reicheren dieser Länder haben in den letzten zwanzig Jahren ein vergleichsweise hohes, die ärmeren Staaten ein relativ schwaches Wachstum aufgewiesen. Schon anhand dieser wenigen Zahlen hebt sich die Kategorie der neuen ostasiatischen Industrieländer, zu denen nebst Korea, Hongkong und Singapur auch Taiwan zu zählen ist, deutlich von den nachstossenden Schwellenländern Malaysia und Thailand ab.

\section{Diversität und Gemeinsames}

Sehr schematisch betrachtet, hat die wirtschaftliche Entwicklung Ostasiens in drei aufeinanderfolgenden Wellen stattgefunden: Die erste Welle stellte das rasche Wachstum Japans in den sechziger Jahren dar, als Nippon zweistellige Zuwachsraten erzielte (heute liegen diese bei "normaleren" 3-4\% pro Jahr). Die siebziger Jahre brachten die zweite Entwicklungswelle mit dem rasanten Aufschwung der neuen Industrieländer Hongkong, Singapur, Südkorea und Taiwan, welche trotz der durch die beiden Ölschocks verursachten Rezession einen rascheren Wachstumsrhythmus aufrechtzuerhalten vermochten als die traditionellen Industriestaaten. Eine dritte Welle ist mit den Entwicklungen in den übrigen ASEAN-Ländern und in China zu erwarten. Positives Element in den Wirtschaftsperspektiven dieser Region ist deren im Vergleich zu anderen Entwicklungs- und Schwellenländern im allgemeinen tragbar erscheinende Schuldenlast, welche in anderen geographischen Räumen schwarze Schatten in die absehbare Zukunft vorauswirft.

Der internationale Handel stellte in mehreren der ostasiatischen Entwicklungsländer einen dominierenden Wachstumsfaktor dar. Von den "Erfolgsländern" Korea, Taiwan, Hongkong und Singapur hat eine ganze Region den Ruf abgeleitet, beispielhaft für wirkungsvolle wirtschaftliche Entwicklungspolitik zu stehen. Die Begünstigung privater Investitionen, die aktive Förderung von exportorientierten Industrien und günstige Kosten- und Lohnverhältnisse haben diesen Ländern zu einem beeindruckenden Exportboom verholfen. Die nachfolgende Tabelle zeigt die erstaunliche Zunahme der Bedeutung von Waren- und Dienstleistungsexporten ausgewählter Länder auf: 
Anteil der Ausfuhr von Waren- und Dienstleistungsexporten am Bruttoinlandsprodukt, in Prozent (4)

\begin{tabular}{lrr|} 
& 1965 & 1985 \\
Hongkong & 71 & 106 \\
Südkorea & 9 & 36 \\
Malaysia & 42 & 55 \\
Thailand & 18 & 27 \\
Philippinen & 17 & 22 \\
Indonesien & 5 & 23 \\
China & 4 & 11 \\
Indien & 4 & 6 \\
Burma & 14 & 6 \\
& & \\
Dazu als Vergleich: & & \\
& & 15 \\
Japan & 11 & 7 \\
USA & 5 & 39 \\
Schweiz & 29 & \\
\hline
\end{tabular}

Es zeigt sich ferner, dass in den meisten ostasiatischen Entwicklungsländern die z.T. rasante Exportsteigerung von der verarbeitenden Industrie ausging und dass der Anteil von Rohwaren an der Gesamtausfuhr dort rückläufig war:

Anteil verarbeiteter Industriewaren am gesamten Warenexport, in Prozent (4)

Singapur

Hongkong

Südkorea

Malaysia

Thailand

Philippinen

Indonesien

China

Indien

Burma
1965

1985

34

87

59

92

81

6

27

35

51

6

11

$\begin{array}{ll}4 & 54\end{array}$

49

49

0 
Von entscheidender Bedeutung für das handelsinduzierte Wachstum der typischen ostasiatischen Exportwirtschaften war - und ist nach wie vor - der Zugang zum amerikanischen Markt. Die USA absorbieren an die $60 \%$ der industriellen Exportprodukte der Entwicklungsländer der Region, und verschiedene dieser Staaten sind für ihre Gesamtausfuhr bis zu 50\% auf die USA angewiesen, mit welchen sie zudem die bilateralen Handelsüberschüsse auftürmen, die zur Finanzierung ihrer eigenen Importbedürfnisse unabdingbar gewesen sind. Die einseitige Exportabhängigkeit bildet das markanteste Faktum der Handelsströme und - wie später zu verdeutlichen sein wird - der Handelspolitik dieser Gegend.

Eine genauere Betrachtung der bilateralen Handelsbilanzen der ASEANLänder (Indonesien, Malaysia, Philippinen, Singapur, Thailand und Brunei) zu anderen Regionen der Welt ist illustrativ: 1986 haben die ASEAN-Staaten mit den USA einen Handelsüberschuss von 3,7 Milliarden US\$ (Exporte: 13,8 Mia \$; Importe: 10,1 Mia \$), mit Japan hingegen einen Überschuss von 1,2 Milliarden US\$ (Exporte: 15,0 Mia \$; Importe: 13,8 Mia \$) erwirtschaftet. Der Handel mit der EG war praktisch ausgeglichen (Exporte: 8,8 Mia \$; Importe: 8,7 Mia \$), und mit dem Rest der Welt wurde ein Defizit von 2,2 Milliarden US\$ (Exporte: 18,0 Mia \$; Importe: 20,2 Mia \$) erzielt. Der Handel unter den ASEAN-Ländern selbst macht weniger als einen Fünftel ihres gesamten Aussenhandels aus (5).

Um den Platz, den sich gewisse ostasiatische Entwicklungsländer unter den Welthandelsnationen erobert haben, zu dokumentieren, sei noch eine letzte Zahlenreihe angeführt: Das GATT-Sekretariat hat im Frühjahr aufgrund der Handelsdaten der letzten drei erfassten Jahre eine "Rangliste" der GATT-Vertragsparteien (6) gemäss deren Anteil am Welthandel errechnet: 1. EG $(40,4 \%)$, 2. USA $(16,8 \%)$, 3. Japan $(9,2 \%)$, 4. Kanada $(5,0 \%)$, 5. Schweiz $(1,9 \%), 6$. Korea $(1,8 \%)$, 7. Hongkong $(1,8 \%), 8$. Schweden $(1,8 \%), 9$. Australien $(1,4 \%), 10$. Österreich (1,2\%), 11. Brasilien (1,2\%), 12. CSSR $(1,1 \%)$, 13. Norwegen $(1,0 \%)$, 14. Singapur $(1,0 \%)$, 15. Indonesien $(1,0 \%), 16$. Mexiko $(0,9 \%), 17$. Südafrika $(0,9 \%)$, 18. Finnland $(0,8 \%)$, 19. Malaysia $(0,8 \%)$, 20. Indien $(0,7 \%)$. Es folgt ferner auf Platz 27 auch Thailand.

Alle diese Statistiken heben die Bedeutung einer auf den internationalen Handel angewiesenen Industrialisierung als bewusstes und erklärtes Entwicklungsziel hervor. Dies stellt eine Konstante aller dieser Länder dar, die gezwungenermassen in der Diversifikation aus dem Primärsektor Wirtschaftswachstum und genügend Beschäftigungsmöglichkeiten für eine nach wie vor zunehmende Bevölkerung suchen müssen. Mit Ausnahme der Stadtstaaten Hongkong und Singapur bauten alle ostasiatischen Entwicklungsländer ihre industrielle Zukunft auf einem dominierenden Landwirtschafts- und Primärsektor auf, welcher traditionell durch binnen- und lokalorientiertes Handwerk und Kleinindustrie ergänzt wurde. Moderne Industrialisierung ist für diese agrarischen Gesellschaften ein neues, junges Phänomen, dessen Ausbreitung und Integration in den internationalen Güterkreislauf je nach Land sehr unterschiedliche Dimensionen und Eigenschaften annimmt. Ausgangsbedingungen und die Notwendigkeit der "Offenheit" stellen sich für kleine, rohstoffarme Länder wie die beiden 
Stadtstaaten, aber auch Südkorea und Taiwan anders dar als für die rohstoffreichen übrigen ASEAN-Staaten und sind grundlegend verschieden von den Verhältnissen in den Grossmächten subkontinentalen Ausmasses (China und Indien, in gewisser Hinsicht auch Indonesien) mit ihren riesigen Binnenmärkten. Man kann also die reiche Vielfalt des ostasiatischen Raumes, die sich etwa in der geographisch konzentrierteren Zone der ASEAN-Länder in faszinierender Weise zeigt, nicht genug herausstreichen.

\section{Der bilaterale Handel: Fakten}

Welche Rolle spielt nun der bilaterale Handel zwischen der Schweiz und den ostasiatischen Entwicklungsländern. Die Handelsbeziehungen unseres Landes mit dem Fernen Osten haben eine lange und interessante Geschichte. Noch heute bestehende Handelshäuser wie Diethelm, Züllig, Berli Jucker und andere, die sich schon im letzten Jahrhundert in Asien niedergelassen hatten, begleiteten schweizerische Manufakturwaren in den Fernen Osten und waren tragende Pfeiler unseres Aussenhandels in der "Pionierzeit". Der Warenaustausch mit den ostasiatischen Entwicklungsländern, insbesondere dem kolonisierten südostasiatischen Raum, wickelte sich insgesamt ganz nach dem überkommenen Schema Industriewaren aus Europa gegen Rohwaren (Gewürze, Kautschuk, Palmöl, Zinn, Edelmetalle usw.) aus dem Fernen Osten ab. Heute basiert der Warenverkehr auf dem Austausch von industriellen Gütern. Dies ist ganz besonders der Fall für die Schweiz, die ihren bescheidenen Bedarf an Rohwaren ohnehin mehrheitlich durch Käufe bei europäischen Erstimporteuren oder ersten Verarbeitern deckt. Die industrielle Arbeitsteilung und die zunehmende Auslagerung von Produktionsstätten mittels Direktinvestitionen relativieren ferner die Aussagekraft der blossen Handelszahlen als Massstab der wechselseitigen Wirtschaftsbeziehungen immer mehr. Die Umsätze, welche namentlich die schweizerischen Grossfirmen der Nahrungsmittel-, der chemisch-pharmazeutischen, aber auch die Unternehmen der Maschinenindustrie über ihre lokalen Tochtergesellschaften erzielen, sind bedeutend (7). Hinzu kommen die zunehmenden Aktivitäten schweizerischer Dienstleistungsunternehmen, insbesondere des Finanzbereichs, die ohnehin schwer bezifferbar und vergleichbar sind.

Quantitativ spielt der Handel zwischen der Schweiz und den ostasiatischen Entwicklungsländern eine sicher bescheidene Rolle. In wenigen dieser Länder dürfte der Anteil schweizerischer Einfuhren am Gesamtimport die 1-ProzentMarke übersteigen. Dasselbe gilt in umgekehrter Richtung, indem nur der Import aus Hongkong in der schweizerischen Handelsstatistik mit einem Anteil von über einem Prozent an der Gesamteinfuhr ausgewiesen wird. Bevor diese Zahlen aber weiter verfolgt werden, lohnt sich selbst für den eiligen Leser ein kurzer Blick auf die eigentliche Substanz dieses Wirtschaftsverkehrs. Listet man alle schweizerischen Einfuhren (nach Tarifnummern) auf, die im vergangenen Jahr (1987) den Wert von 5 Millionen Franken je Herkunftsland überschritten haben, gelangt man zu einer recht langen Aufzählung, die eine erstaunlich breite $\mathrm{Pa}$ - 
lette von verschiedensten Warengattungen umfasst: Die Schweiz kauft von den hier betrachteten Entwicklungsländern:

Fischzubereitungen und -konserven (Taiwan, Thailand), Kaffee (Indonesien, Indien), Früchte (Philippinen), Gemüse (China, Indien), Tabak (Indonesien), Zinn (Malaysia), chemische Stoffe (Südkorea, Taiwan, Thailand), Reiseartikel und Taschen aus Stoff (Hongkong, Südkorea, Taiwan, China, Indien), Lederwaren (Hongkong, Südkorea, China), Teppiche (China, Indien), Seide und Seidengewebe (China), Kleiderwaren (Hongkong, Südkorea, Taiwan, Thailand, China, Indien), Schuhe (Südkorea, Taiwan), Edelsteine und Schmucksteine (Singapur, Hongkong, Thailand, Indien), Bijouterie- und Juwelierwaren (Hongkong, Thailand), Nähmaschinen und -nadeln (Taiwan), Werkzeugmaschinen für die Metallverarbeitung (Taiwan), Statistik- und Lochkartenmaschinen (Singapur, Hongkong, Taiwan), elektrische Transformatoren usw. (Hongkong), Sende- und Empfangsgeräte (Singapur, Hongkong, Südkorea, Taiwan, Malaysia), Spannungsregler und anderes elektrisches Gerät (Taiwan), Elektronenröhren, Photozellen usw. (Hongkong, Taiwan), Uhren und Uhrenbestandteile (Hongkong, Malaysia, Thailand), Tonaufnahme- und Tonwiedergabegeräte usw. (Singapur, Hongkong, Südkorea), Spielzeug (Hongkong, Südkorea, Taiwan) und Sportartikel (Taiwan).

Allgemein widerspiegelt sich im Verarbeitungsgrad der importierten Güter das Niveau des industriellen Entwicklungsstandes des jeweiligen Exportlandes: einfache Landwirtschaftsprodukte stammen aus den relativ ärmeren Entwicklungsländern, die Maschineneinfuhren fast ausschliesslich aus den vier neuen Industriestaaten. Kleiderwaren werden (noch) aus zahlreichen der betrachteten Länder eingeführt; dasselbe trifft auf Uhren zu, wobei aus Hongkong vor allem Fertiguhren, aus Malaysia und Thailand hingegen Uhrenbestandteile stammten. Interessant sind schliesslich einige traditionelle "Spezialitäten" wie z.B. die chinesische Seide.

Trotz der Breite des Warensortiments konzentrieren sich die schweizerischen Importe aus einigen Herkunttsländern aber auf jeweils enge Segmente: Hongkong liefert in überwiegendem Masse Kleider und Schmuckwaren, in Korea nehmen ebenfalls Kleider sowie Sende- und Empfangsgeräte einen dominanten Platz ein. Taiwans wichtigstes Exportprodukt nach der Schweiz waren Statistik- und Lochkartenmaschinen, und Chinas bedeutendste Ausfuhr bildeten Kleiderwaren.

Was liefert die Schweiz? Es findet sich ebenfalls ein ansehnlicher Ausschnitt aus der Palette schweizerischer Industrieprodukte in den Exporten nach den ostasiatischen Entwicklungsländern, insbesondere nämlich Maschinen, elektrische Geräte, Uhren, Schmuck- und Juwelierwaren, sowie einige Chemikalien und Lebensmittel. Wie bereits enwähnt, sind die schweizerischen Nahrungsmittel- und Chemiefirmen lokal gut vertreten und dürtten ihre ausländische Produktion vorwiegend aus Drittlandquellen alimentieren. Gemäss Handelsstatistik exportierte die Schweiz 1987 folgende Waren mit einem Wert von über 5 Millionen Franken pro Destination: 
Zuckerwaren und Schokolade, chemische Erzeugnisse, Vitamine, Arzneiwaren, Farbstoffe, Riech- und Aromastoffe, Desinfektions- und Schädlingsbekämpfungsmittel, Baumwollgewebe, Schuhe, Edel- und Schmucksteine, Gold und Goldlegierungen, Bijouterie- und Juwelierwaren, Sägen, Dampfkessel, Pumpen, Apparate zur thermischen Behandlung von Stoffen, Hebeund Fördermaschinen, Müllereimaschinen, Papiermaschinen, Druckereimaschinen, Spinnereimaschinen, Webstühle, Strick- und Stickmaschinen, Maschinen zur Spinnstoffbehandlung, Giessmaschinen, Werkzeugmaschinen, verschiedene andere Maschinen, Armaturen für Leitungen, Transmissionswellen und Getriebe usw., elektrische Generatoren und Motoren usw., elektromechanische Handwerkzeuge, elektrische Industrieöfen, Telephonund Telegraphieapparate, Sende- und Empfangsgeräte, Strombrecher und Spannungsregler usw., elektrische Lokomotiven, Elektronenröhren und Photozellen usw., medizinische Apparate und Instrumente, Mess- und Regulierrinstrumente, Uhren, Uhrwerke und andere Uhrenfurnituren, Bilder und Zeichnungen.

"Grosse Posten" (mit einem Wert von über 50 Mio Franken je Land) waren 1987 die Lieferung von Farbstoffen und Edel- und Schmucksteinen nach Hongkong, von Textilmaschinen nach Taiwan und China, von Werkzeugmaschinen und elektrischen Geräten nach China sowie von Uhren und Uhrenbestandteilen nach Singapur, Hongkong, Taiwan und Thailand. Volumenmässig schnell einmal ins Gewicht fallen in diesen Handelsbeziehungen einzelne Grossaufträge, wie beispielsweise die Lieferung von neun Lokomotiven an China (34,7 Mio Fr.). Insgesamt sind die traditionellen Schweizer Industriewaren, insbesondere die Erzeugnisse der Maschinenindustrie, aber recht breit im gesamten Exportbild gestreut.

Betrachtet man den Handel der Schweiz mit den ostasiatischen Entwicklungsländern in seinen quantitativen Ergebnissen, stellt man grosse Schwankungen - sowohl import- wie exportseitig - von Land zu Land fest. Das Gesamtresultat darf sich aber sehen lassen, und die allgemeine Entwicklung der letzten zehn Jahre, von einem teilweise allerdings sehr bescheidenen Niveau ausgehend, ist durchaus als überdurchschnittlich zu bezeichnen. Die im Anhang befindliche Tabelle stellt einige Daten für die Vergleichsperiode 1977-1987 zusammen.

Das gesamte Handelsvolumen der Schweiz mit den aufgelisteten asiatischen Entwicklungsländern belief sich 1987 auf knapp 7 Milliarden Franken, was rund 5 Prozent unseres gesamten Aussenhandels entsprach. Diese Zahlen muten sicher bescheiden an, sind aber im Vergleich zum Warenaustausch mit Afrika (3 Mia Fr.) und Lateinamerika (4 Mia Fr.), ja selbst mit Japan (6 Mia Fr.) und Nordamerika (11 Mia Fr.) recht beachtlich. Durchaus bemerkenswert ist hingegen die Feststellung, dass mit Ausnahme der Philippinen alle diese Länder als Absatzmärkte für Schweizer Waren in den letzten zehn Jahren überdurchschnittlich gewachsen sind; die Versechsfachung unserer Lieferungen nach Taiwan, die Steigerung um 356\% unserer Ausfuhren nach China sind aus- 
sergewöhnlich. Aber auch in die übrigen Länder hat sich der Export zwei- bis dreimal so schnell entwickelt wie der gesamte schweizerische Aussenhandel. Importseitig ist das Bild weniger einheitlich: bei im allgemeinen überdurchschnittlichen Zunahmen der Einfuhren - insbesondere aus Südkorea und Taiwan - fällt der Rückgang der Lieferungen aus einigen Ländern seit 1977 auf. Gesamthaft gesehen zeichnete sich der schweizerische Handel mit der Region - Japan übrigens eingeschlossen - durch eine Dynamik aus, welche sowohl export- wie importseitig besteht und sich deutlich vom Güterverkehr mit den anderen Regionen der Dritten Welt abhebt. Aus handelspolitischer Perspektive ein überraschendes Faktum stellen die positiven Handelsbilanzen der Schweiz auch mit den jungen Industrieländern - dar, eine im Vergleich zu anderen traditionellen Industrieländern eher ungewöhnliche Situation. Einzig mit Südkorea entstand 1987 ein kleiner Fehlbetrag. Mit den "vier Tigern" wurde gesamthaft ein Überschuss von rund einer Milliarde Franken erwirtschaftet - genug, um das Defizit gegenüber Japan abzudecken. Diese Resultate - dies sei hier vorweggenommen - sind unter Bedingungen des offenen Marktzugangs auf schweizerischer Seite erzielt worden, wiederum eine im Vergleich zu anderen traditionellen Industrieländern durchaus ungewöhnliche Situation.

\section{Der bilaterale Handel: Sachprobleme}

Gemessen an der relativ grossen Dichte der Wirtschaftsbeziehungen zwischen der Schweiz und den ostasiatischen Entwicklungsländern halten sich die auftretenden bilateralen Sachprobleme im "Rahmen des Normalen". Weder hüben noch drüben wird die Reizschwelle leicht überschritten, so dass die Führung der Wirtschafts- und Handelspolitik sich bisher nach den angestammten, klassischen Mustern ausgerichtet hat. Der interessierte Leser findet in den alljährlich vom Bundesrat ans Parlament gerichteten Aussenwirtschaftsberichten eine umfassende Orientierung über die gesamte Aussenwirtschaftspolitik, einschliesslich die konkreten handelspolitischen Aktionen, die jeweils im Verhältnis zu den Ländern der verschiedenen Regionen von schweizerischer Seite her unternommen worden sind. So beschränken sich denn die schweizerischen Anliegen in Ostasien nicht bloss auf den Abbau der Importhemmnisse an den Grenzen (Zölle und mengenmässige Beschränkungen), die schweizerischen Exporten den Marktzutritt erschweren oder verunmöglichen, auch interne Massnahmen (z.B. Preisvorschriften) bilden je länger je mehr Gegenstand bilateraler Gespräche und Verhandlungen. Bezüglich Investitionen findet im ostasiatischen Raum ebenfalls die Politik der Absicherung von Direktinvestitionen durch bilaterale Investitionsschutzabkommen Anwendung; besonders zu erwähnen ist in diesem Zusammenhang die Unterzeichnung eines solchen Vertrages mit China im November 1986, welcher mittlerweile in Kraft getreten ist. Die an die ärmeren dieser Länder verliehenen Mischkredite, welche aus entwicklungspolitischen Gründen wichtige Vorhaben mitfinanzieren, gehören ebenfalls zu den traditionellen Instrumenten der bilateralen Aussenhandelspolitik (8). Aktuell geworden 
ist mit den Zahlungsbilanzschwierigkeiten der Philippinen auch in dieser Region das Thema der Schuldenkonsolidierung: In zwei bilateralen Abkommen, die auf entsprechenden Übereinkommen im Rahmen des Pariser Klubs basieren, mussten die Fälligkeiten aus Philippinen-Krediten seit 1985 umgeschuldet werden. Ein Problem, das in letzter Zeit mit verstärkter Intensität behandelt wird, bilden der mangelnde Patentschutz (besonders auf Pharmazeutika und Chemikalien) sowie die an grosser Zahl auftretenden Fälschungen schweizerischer Qualitätsprodukte. Die bilaterale Handelspolitik, welche die Schweiz in der Region führt, hat sich also eine volle, dem Entwicklungs- und Industrialisierungsstand der einzelnen Handelspartner entsprechende Agenda von Sachproblemen vorgenommen.

Was die Begehren in umgekehrter Richtung, d.h. an die Adresse der Schweiz gerichtet, anbelangt, wird unserem Land eine offene Handelspolitik im Industriewarenbereich durchaus attestiert. Im Gegensatz zu praktisch allen industrialisierten Staaten hat die Schweiz keine handelsbeschränkenden Arrangements mit den konkurrenzfähigen Exporteuren aus Fernost vereinbart, und die Zollschranken und übrigen Einfuhrmassnahmen, welchen Industriewaren an der schweizerischen Grenze unterworfen sind, halten sich in einem vergleichsweise bescheidenen Rahmen. Wenig erbaut sind hingegen die Agrarexportländer über das Ausmass des schweizerischen Landwirtschaftsprotektionismus; die durch hohe Stützungspreise und Importschranken gesicherte Erhaltung oder sogar Neueinführung von Kulturen in der Schweiz, für deren Anbau u.a. gewisse ostasiatische Entwicklungsländer natürliche Kostenvorteile haben, ist nicht gerade dazu angetan, unseren eigenen handelspolitischen Begehren Rückhalt zu geben und unserem Eintreten für eine liberale Welthandelsordnung Nachhalt zu verschaffen.

Bevor dieser faktische Beschrieb der bilateralen Beziehungen der Schweiz mit den ostasiatischen Entwicklungsländern und der wichtigsten handelspolitischen Sachprobleme abgeschlossen ist, sind noch einige grundsätzliche Anmerkungen zur bilateralen Handelspolitik als solcher vonnöten. Die bilaterale Handelspolitik eines kleinen Landes - sei dies im Umgang mit einer industrialisierten Grossmacht wie den USA oder im Verhältnis mit kleineren oder weniger entwickelten Staaten - greift in der Regel klar begrenzte Sachprobleme auf, die oft auch einer konkreten Lösung zugeführt werden können. In diesem Sinne ist bilaterale Handelspolitik sehr "business-like". Es wird über Zölle, Kontingente, Preise, Lizenzen, Investitionsvorschriften, Kredite usw. verhandelt. Idealerweise können sich solche Verhandlungen an allgemeinen Prinzipien und Regeln orientieren, welche in multilateralen Fora, in welchen die beiden Partner vertreten sind, bereits ausgehandelt und fixiert worden sind. Die multilaterale Wirtschaftszusammenarbeit sollte in diesem Sinne die Gefässe bereitstellen, in denen der bilaterale "contentieux" eingefangen und bewältigt werden kann.

Ein funktionierendes multilaterales System bietet auch Gewähr dafür, dass die konkrete bilaterale Ausmarchung nicht zu einem blossen Kräftemessen wird, in welchem der wirtschaftlich oder politisch Schwächere unweigerlich den kürzeren ziehen muss. Denn der Schwächere wird im multilateralen Vorgehen dop- 
pelt geschützt: Einerseits kann er nur im Zusammenschluss mit einer signifikanten Anzahl anderer Partner hoffen, einer Grossmacht gegenüber wirkungsvoll auftreten und seine Interessen durchsetzen zu können. Andererseits stellen verbindliche multilaterale Regeln "Bandbreiten" dar, innerhalb derer sich die Grossmacht in der Verfolgung ihrer Interessen zu bewegen hat. Werden diese multilateralen Regeln nicht mehr eingehalten, oder existieren sie in einem gegebenen Bereich überhaupt nicht, gieicht die bilaterale Verhandlung einem Zweikampf ohne übergeordnete Spielregeln. Die zum Teil extrem hohe Abhängigkeit der ostasiatischen Exporteure vom amerikanischen Markt erklärt deren konstante, überwiegende Sorge um ihr Verhältnis zu den USA, welches natürlich nicht nur aus rein handelspolitischer oder wirtschaftlicher Sicht, sondern auch aus politisch-militärischer Perspektive für die Region von existentieller Bedeutung ist. Zumindest in Handelsfragen darf man in letzter Zeit ohne Übertreibung von einer mentalen Fixation auf Verhalten und Launen eines unkontrollierbaren und unvorhersehbaren amerikanischen Kongresses sprechen; dies ist verständlich. Ebenso prioritär, so würde man folgern, müsste logischerweise das Interesse dieser Länder an multilateralen Regeln sein, wie sie in umfassendster Weise im Allgemeinen Zoll- und Handelsabkommen (GATT) und seinen annexen Übereinkommen niedergelegt sind, sowie - ganz allgemein - an einem als Institution funktionierenden und international respektierten GATT.

\section{Strapaziertes Handelssystem}

"Der Anstieg der Fertigwarenausfuhren der Entwicklungsländer und die Steigerung ihres Anteils an den Fertigwarenausfuhren der entwickelten Länder und der Entwicklungsländer zusammengenommen von einem Zwanzigstel 1963 auf fast einen Siebtel 1984 war die wichtigste Veränderung betreffend die Handelsströme in der Nachkriegszeit", meint der bekannte Ökonom Bela Balassa (9). Die Anschlussfrage, die sich aus handelspolitischer, bzw. institutioneller Sicht sofort stellt, lautet: Wie hat das Handelssystem diesen radikalen Wandel verkraftet? Die Antwort lautet: schlecht. Deutlichstes Indiz dafür sind die unzähligen Abmachungen ausserhalb des GATT in Form von Exportbeschränkungsabkommen, sogenannten "orderly marketing agreements", Exportvorgaben, Preisüberwachungen, intra-industriellen Arrangements, diskriminatorischen Importsystemen usw., welche vor allem den internationalen Güterverkehr in Stahl und Stahlprodukten, Werkzeugmaschinen, Automobilen, Elektronik, Schuhen, Textilien und Bekleidung (innerhalb und ausserhalb des im GATT ausgehandelten Multifaserabkommens) sowie Landwirtschaftsprodukten und Nahrungsmitteln weitgehend "organisiert". Das im Prinzip einzige permanente Schutzinstrument, welches das GATT erlaubt, - nämlich der Zoll - und die ebenfalls im GATT vorgesehenen "escape clause"-Mechanismen zur temporären Abfederung plötzlichen Importdrucks - vermochten offensichtlich keine situationsgerechten Lösungen anzubieten. Die Flucht in die handelspolitische "Grauzone" wurde, allen überzeugenden ökonomischen Gegenargumenten zum 
Trotz, zum handelspolitischen Standardverhalten der letzten zehn Jahre. Es mag zutreffen, dass mehrere solcher Abkommen anstelle von legitimen unilateralen Abwehrmassnahmen gegen unlautere Konkurrenz (Anti-Dumping- und Ausgleichszollmassnahmen) eingerichtet wurden. Das jüngste (und sicher nicht vollständige) Verzeichnis von in Kraft stehenden Grauzonen-Abmachungen, welches das GATT-Sekretariat zusammengestellt hat, zeigt aber mit aller Deutlichkeit, dass auf diesem Wege in erster Linie die sich in den Industriestaaten stellenden sektoriellen Strukturprobleme beantwortet wurden. Es wimmelt in diesem Verzeichnis nur gerade von Abkommen mit Korea und Taiwan, und auch Singapur und Thailand sind gut vertreten (10). In der Liste der Exporteure mit bilateralen Abkommen unter dem multilateralen Textilabkommen (MFA) finden sich alle ostasiatischen Entwicklungsländer. Neben eigentlicher "Grauzone" und MFA zeigt auch die immer laxere - und willkürliche - Auslegung der Dumping- und Subventionsregeln, wie sehr die Balken des Welthandelssystems unter dem Druck eines fundamental veränderten internationalen Wettbewerbs knarren.

Wenn somit die GATT-Konformität eines wichtigen Teils der handelspolitischen Instrumentarien und Praktiken in Zweifel gezogen werden muss, so ist es um das vom heutigen GATT vorgegebene Gleichgewicht zwischen Rechten und Verpflichtungen der einzelnen Vertragsparteien nicht minder zweifelhaft bestellt. Dass Entwicklungsländer auf dem schwierigen Weg der Industrialisierung und der Eingliederung in eine bessere internationale Arbeitsteilung sich nicht einfach denselben handelspolitischen Spielregeln unterwerfen können, welche die Industriestaaten in der Wiederaufbauphase nach dem Zweiten Weltkrieg - für sich - aufgestellt haben, ist unbestritten. Jede noch so oberflächliche Betrachtung der gesellschaftlichen und politischen Realitäten in der Dritten Welt macht dies evident. Dass die neuen, erfolgreichen Industrieländer in ihren Beziehungen zu den traditionellen Industriestaaten einen permanenten Freipass in der Gestaltung ihrer eigenen Handelsregimes haben sollen, stellt für letztere aber eine politische Unmöglichkeit dar. Überdurchschnittlich hohe Zollsätze; die Freiheit, diese nach Belieben modifizieren zu dürfen; geringe bis nichtexistente multilaterale Disziplin in der Administration nichttarifarischer Importbarrieren; ein dem internationalen Wettbewerb entzogenes öffentliches Beschaffungswesen; die Möglichkeit, mittels Einfuhrquoten die Handelsströme zu lenken und gleichzeitig massive Devisenreserven aufzuhäufen usw., - all dies stellt eine handelspolitische Spezial- und Vorzugsbehandlung dar, welche die starkem protektionistischem Druck von Innen ausgesetzten Regierungen der "alten" Industriestaaten politisch nicht auszuhalten vermögen. Das Gleichgewicht zwischen Rechten und Verpflichtungen ist gestört. Die Verbesserung der GATT-Disziplin der traditionellen Industriestaaten und die Übernahme gewisser internationaler Verpflichtungen durch die jungen Industrieländer bedingen sich gegenseitig, stellen eine wirtschaftliche und politische Notwendigkeit dar. Es ist dies ein zentrales Anliegen und ein schliesslicher Erfolgsmassstab für die im GATT derzeit stattfindende achte Welthandelsrunde, die sogenannte "Uruguay-Runde". Es wird sich nicht zuletzt in diesen Verhandlungen weisen müssen, ob der Übergang von 
einem von den traditionellen Industrieländern für sich selbst geschaffenen Handelssystem zu einer wirklich umfassenden multilateralen Welthandelsordnung, welche neben dem Güterverkehr auch den internationalen Dienstleistungsaustausch einschliesst, gelingen kann. Die Schweiz würde darin auch ihre eigenen aussenwirtschaftlichen Interessen am besten gewahrt sehen.

\section{Vielfältige Interessenlagen}

Nichts wäre aber kurzsichtiger und unproduktiver, als die Reform des multilateralen Handelssystems als Ausmarchung zwischen Interessen zu sehen, die einem simplen und alles durchziehenden Nord-Süd-Gegensatz entspringen. Gerade der ostasiatische Raum zeigt exemplarisch, wie sehr eine derartige Sicht der Dinge wirklichkeitsfremd und schablonenhaft ist, auch wenn sie in den internationalen Gremien, aus meistens verhandlungstaktischen Gründen, immer noch des öfteren vorgeschoben wird. Der ostasiatische Raum bietet sozusagen ein kontinuierliches Spektrum von nationalen wirtschaftlichen Interessen dar, welches auch unter den Entwicklungsländern zu sehr ungleichen Perzeptionen der eigenen Verhandlungsposition (beispielsweise in der Uruguay-Runde) führen kann. Illustrativ dafür mögen die grundsätzlichen Zielsetzungen zweier dieser Staaten in den GATT-Verhandlungen stehen:

Das eine Land, am unteren Ende der Einkommensskala stehend, sieht sich selbst als in einem schwierigen Entwicklungs- und Industrialisierungsprozess engagiert, dessen Autonomie und Unabhängigkeit vor Einmischung der industrialisierten Staaten bewahrt werden muss. Das GATT stellt in diesem Sinne in erster Linie ein Instrument dar, welches unilaterale Eingriffe der westlichen Industriemächte zügeln soll. Die Offensivinteressen dieses Landes liegen vornehmlich im Bereich der Textilien, in welchem die Exportmöglichkeiten durch die im Norden verhängten Einfuhrquoten schmerzlich beschränkt sind, sowie auf dem Gebiet der Landwirtschaft. Die Autonomie der nationalen Agrarpolitik, und damit des eigenen Agrarhandels, darf jedoch nicht tangiert werden, denn Versorgung der Bevölkerung mit Nahrungsmitteln und Finanzierung der Industrialisierung aus dem in der Landwirtschaft erwirtschafteten Überschuss sind "overriding national objectives". Vielmehr geht es darum, den Zugang für Agrargüter, insbesondere für tropische Produkte, zu den Märkten im Norden zu verbessern. Subventionen sind ein zentrales Instrument der Wirtschaftspolitik in Entwicklungsländern und können nicht untersagt werden; die Gegenmassnahmen der Industrieländer in Form von Anti-Dumping- und Ausgleichszöllen sollten hingegen strikteren multilateralen Regeln unterworfen werden. Auch die Möglichkeit temporären Importschutzes, unter den "escape clauses", sollten verbindlicher geregelt werden. Das eigene Handelsregime wird auf Geheiss der internationalen Finanzinstitutionen bereits "rationalisiert", und die vermehrte Bindung von Zöllen ist denkbar. Abgaben auf Importen und Exporten haben aber weniger eine handelspolitische als eine fiskalische Funktion, indem weit mehr als die Hälfte aller Regierungseinnahmen aus dieser Quelle stammen. Investi- 
tionsbestimmungen gehören in die Domäne nationaler Hoheit, multilateralen Regeln über Dienstleistungen wird skeptisch begegnet und strengerer Schutz des geistigen Eigentums als Protektionismus des Nordens betrachtet.

Den zweiten Fall bildet ein Land am oberen Ende der Einkommensskala: Obwohl wirtschaftlich stark von den USA abhängig, sieht dieses Land sein Interesse nicht allein in der Sicherung des Marktzugangs im Norden, sondern - und dies ist ein grundlegender Unterschied zum erstgenannten Fall - auch in der Öffnung anderer Entwicklungsländer für seine Güter- und Dienstleistungsexporte sowie für zukünftige Direktinvestitionen. Ebenfalls im Gegensatz zum erstbeschriebenen Fall hat sich dieses Land recht gut mit den von den Industrieländern des Nordens verhängten Textileinfuhrquoten arrangiert, die ihm garantierte Marktanteile und höhere Preise sichern. Bezüglich der Landwirtschaft (inklusive tropische Produkte) sind die Interessen durchwegs defensiv: ein kostspieliger Agrarprotektionismus stellt das Überleben des Bauernstandes und einen minimalen Selbstversorgungsgrad sicher. Die Einschränkung des AntiDumping- und Ausgleichszollinstrumentariums stellt im Hinblick auf die Gefahr unilateraler Anwendung durch die "Grossen" eine erstrangige Zielsetzung dar. Eine verbindliche Regelung der "escape clause"-Möglichkeiten ist wichtig im Sinne der Sicherung des Marktzugangs im Norden; die verschiedenen Grauzonenvereinbarungen stellen jedoch sekundäre Probleme, solange die Mitkonkurrenten (insbesondere aus Fernost) nicht grössere Einfuhrquoten in Amerika und Europa erhalten. Das eigene Importregime wird auf äusseren Druck hin bereits liberalisiert, und das Bewusstsein, dass in dieser Hinsicht vermehrte Verpflichtungen unumgänglich sein werden, ist vorhanden. Es besteht ein klares Interesse an liberaleren Investitionsbedingungen und Absatzmöglichkeiten für Dienstleistungen, insbesondere in weniger entwickelten Ländern der Gegend. Die gesetzlichen Bestimmungen über geistigen Eigentumsschutz sind bereits an die amerikanische Legislation angepasst worden, die bestehenden Vollzugsprobleme sollen auf bilateraler Ebene behandelt werden.

Dieser kurze Blick auf die Verhandlungslandschaft der "Uruguay-Runde" zeigt besser als eine Menge Statistiken, wie verschiedene ostasiatische Entwicklungsländer ihre teilweise divergierenden Interessen definieren, wo sie ihren heutigen Platz und ihr zukünftiges Potential im multilateralen Handelssystem sehen. Sie alle über einen Leisten schlagen zu wollen, wäre verfehlt und töricht, denn die Chance, ein der Realität verschiedener Volkswirtschaften angepassteres Welthandelssystem zu schaffen, würde vergeben.

\section{Mehrdimensionale Handelspolitik}

Die multilaterale Handelspolitik ist nur eine Dimension der internationalen Handelspolitik. Sie mag der schweizerischen Konzeption aus systemischen und pragmatischen Gründen am nächsten liegen. Gerade im Verhältnis zum ostasiatischen Raum, insbesondere zu Japan und den neuen Industrieländern, hat sich aber eine mehrdimensionale internationale Handelspolitik in den letzten 
Jahren fest etabliert. Schrittmacher waren einmal mehr die USA, die seit Anfang dieses Jahrzehnts ihre wirtschaftlichen Interessen ganz offen sowohl auf multiwie bilateralen Wegen verfolgen, die sich gegenseitig ergänzen sollen. Das bilaterale Vorgehen hat sich dabei als durchaus wirkungsvoll erwiesen. Taiwan und Südkorea bieten instruktiven Anschauungsunterricht: Die Regierungen dieser beiden Länder wurden sehr handfest mit Begehren nach Handelsliberalisierung konfrontiert, welche im Falle Südkoreas unlängst mit durchschnittlichen 40\%-igen Zollsenkungen auf 800 Produkten, im Falle Taiwans mit einer Halbierung der Zollansätze auf 3600 Positionen beantwortet wurden. Davon sollten nicht nur die überseeischen Lieferanten sondern auch die Konsumenten in diesen beiden Ländern profitieren. Die USA haben ferner massiven Druck auf die Wechselkurspolitik der beiden Regierungen ausgeübt; früher an den Kurs des amerikanischen Dollars gebunden, haben sich der koreanische Won in den letzten zwei Jahren um $20 \%$, die Währung Taiwans um $30 \%$ gegenüber dem amerikanischen Dollar aufgewertet. Die Wirkung auf die bilateralen Handelsströme zeichnet sich bereits deutlich ab, und die Konkurrenzfähigkeit bestimmter Industriezweige in den neuen Industrieländern wird dadurch fundamental verändert, nicht zuletzt zugunsten anderer ostasiatischer Entwicklungsländer wie Thailand oder die Philippinen. "Asia"s own wealth is trickling down" (11).

Das politisch-wirtschaftliche Klima, in welchem derartige wirtschaftspolitische Anpassungen in den ostasiatischen Entwicklungsländern erfolgen, ist oft recht rauh und kann die bilateralen Handelsbeziehungen zeitweise arg strapazieren. Die kürzliche Entscheidung der US-Regierung, die "vier Tiger" von der Liste der Empfänger amerikanischer Präferenzzölle zu streichen, welche den Entwicklungsländern autonom und einseitig gewährt werden, hat hohe Wellen geworfen, ebenso wie die Methode der Europäischen Gemeinschaften, mit analoger Drohung Zugeständnisse auf dem Gebiete des geistigen Eigentumsschutzes zu fordern. Es ist evident, dass die Schweiz angesichts ihres kleinen Marktes mit wenig Erfolg selber zu solchen handelspolitischen Druckmitteln greifen könnte. Eine besondere Herausforderung für die schweizerische Handelspolitik ist deshalb, sicherzustellen, dass die in anderen Ländern - autonom oder weniger autonom - erfolgende Liberalisierung zu keinen Diskriminierungen zugunsten der alleinigen "Grossen" und zum Nachteil der Akteure in Drittstaaten wie der Schweiz führt. Dieser Gefahr kann wiederum am besten durch die Verankerung und Bekräftigung des Prinzips der Nichtdiskriminierung als des tragenden Pfeilers des multilateralen Handelssystems vorgebeugt werden.

Die mehrdimensionale handelspolitische Praxis trägt der Tatsache Rechnung, dass der internationale Wirtschaftsverkehr, der durch eine zunehmende Globalisierung von Produktion und Märkten gekennzeichnet ist, unter Ländern mit verschiedenartigen Wirtschaftssystemen stattfindet. Ein in der "Harvard Business Review" kürzlich erschienener Artikel (12) unterscheidet nach den vier Kriterien (a) des Regierungseinflusses auf das Wirtschaftsgeschehen, (b) der Eigentumsverhältnisse in der Industrie, (c) der Ausrichtung der Wirtschaftspolitik auf entweder Regeln oder Resultate und (d) der handeltreibenden Akteure zwi- 
schen fünf Wirtschaftsmodellen: das auf Freihandel basierende System angelsächsischer Tradition, die zentrale Planwirtschaft, die in Westeuropa verbreiteten "mixed economies", den heterogenen Korb der "developing economies" sowie das planorientierte Modell, welches generell auf Länder wie Japan, Taiwan und Korea zutrifft. Während sowohl im Freihandels- wie im planorientierten System das private Eigentum bestimmend ist, bestehen grundlegende Unterschiede bezüglich der Rolle der Regierungen. Diese stellen im ersteren Falle die "Spielregeln" auf, innerhalb welchen die Wirtschaftssubjekte dann frei disponieren ("rules-oriented"); im zweiten Falle hingegen legen sie gewisse Zielvorgaben für die Wirtschaftstätigkeit selbst fest ("results-oriented"). Es ist die interessante These der beiden Autoren, dass die multilaterale, normative Handelspolitik im Verkehr mit planorientierten Ländern ungenügend ist, ja sogar zum Scheitern verurteilt sein kann. Dem Freihandel verpflichtete Partner verhandeln unter sich über die Eliminierung von Massnahmen, welche die Marktkräfte lähmen (Zölle, nicht-tarifarische Hindernisse, Subventionen usw.); Verhandlungen mit einem planorientierten Partner müssten hingegen auf (quantifizierte) wirtschaftliche Ziele (Marktanteile, zulässige Handelsungleichgewichte, Zusammensetzung des Handels usw.) sowie auf Zeitpläne und Überwachung von deren Implementierung ausgerichtet sein. Die Handelspolitik vor allem der USA und der EG mit planorientierten Ländern - einschliesslich ostasiatischer Entwicklungsländer - entspricht heute, zumindest in weiten Teilen, diesem letzteren Schema: ein Trend, der sich umso länger fortsetzen und umso mehr dominieren wird, je weniger die Integration der neuen Handelspartner in eine offene multilaterale Ordnung gelingt.

\section{Schlussbemerkungen}

Die Entwicklungsländer des ostasiatischen Raumes zeichnen sich durch eine grosse Diversität aus. Das herausstechende Merkmal der neuen Industrieländer Asiens bildet ihre exportgestützte Industrialisierungs- und Entwicklungspolitik, die - verbunden mit einer frappanten Erhöhung der Ersparnisraten - diese Länder innert wenigen Jahren auf die vordersten Ränge der Welthandelsgemeinschaft katapultiert hat. Wie in diesem Artikel beschrieben, macht nun gerade ihr Erfolg wichtige wirtschafts- und handelspolitische Anpassungen in den neuen Industriestaaten erforderlich. Der Abbau von offenen und verdeckten Handelsbarrieren, die Liberalisierung der nationalen Finanzmärkte und Devisenkontrollen, die weitere Anpassung der Wechselkursverhältnisse sowie die Deregulierung gewisser Dienstleistungsbranchen dürften neue und veränderte Wachstumsimpulse geben und ein fortgesetztes "up-grading" der industriellen Strukturen dieser Länder zur Folge haben. Die Rolle solcher Volkswirtschaften innerhalb der internationalen Arbeitsteilung wird sich weiter verändern - und gleichzeitig mehr Raum für die industrielle Entwicklung anderer Entwicklungsländer schaffen. Staaten wie Malaysia und Thailand verfügen bereits über eine beträchtliche industrielle Produktionskapazität, und auch Indonesiens Indu- 
strie, obwohl weniger fortgeschritten, diversifiziert sich zunehmend aus dem dominierenden Ölsektor in andere Wachstumsbranchen. Die Unterschiede zwischen den ostasiatischen Entwicklungsländern nicht nur bezüglich natürlicher Ressourcen, sondern auch hinsichtlich ihrer technologischen und organisatorischen Kräfte könnten die Grundlage für eine wachsende wirtschaftliche Zusammenarbeit bieten, in welcher Japan sicher eine Schlüsselposition einnehmen müsste. Die Öffnung Chinas stellt eine Entwicklung von grösster internationaler Bedeutung dar und wird dieses Land zunehmend zu einem Faktor in der internationalen Rolle Ostasiens werden lassen.

So deuten denn die neuen Ausrichtungen der nationalen Wirtschaftspolitiken und Prioritäten auf die Möglichkeit einer engeren regionalen Wirtschaftszusammenarbeit im ostasiatischen Raum hin, welche natürlicherweise auch zu einer verstärkten Regionalisierung des Handels führen könnte. Es wäre dies eine weitere Bekräftigung einer beinahe weltweit erkennbaren Tendenz Westeuropa und Nordamerika sind die offensichtlichsten Beispiele -, welche das multilaterale Handelssystem auf die Dauer aushöhlen und das GATT letzten Endes in eine gewissermassen "theoretische Existenz" verdrängen könnte. Dieser Tendenz entgegen stehen jedoch im Falle des ostasiatischen Raumes dessen wirtschaftlich-technologische, aber auch weitgehend politischmilitärische Ausrichtung auf die USA sowie die kommerzielle Abstützung des eigenen Wohlstandes auf die grossen Märkte vor allem Nordamerikas, aber auch Westeuropas. Die Direktinvestitionen Japans bezeugen unmissverständlich, wo die wirtschaftliche Zukunft gesehen und gesucht wird: Die japanischen Direktinvestitionen haben sich in den letzten sieben Jahren versiebenfacht, der Anteil Nordamerikas als Empfängergebiet stieg in derselben Periode von $34 \%$ auf $46 \%$, derjenige Westeuropas von $13 \%$ auf $20 \%$, während Asien als Destination - trotz eindrücklichen Zunahmen in absoluten Zahlen - von 35\% auf $18 \%$ zurückfiel.

Die mögliche Tendenz einer von Japan ausgehenden Regionalisierung des Handels der ostasiatischen Entwicklungsländer wird von der transpazifischen Dimension und deren treibender Kraft - der bilateralen Beziehung mit den USA - überlagert. Neben diesem Kräftefeld zwischen Regionalisierung und bilateraler Dominanz werden die Handelsbeziehungen Westeuropas mit dem ostasiatischen Raum ihren Platz definieren. Die Schweiz hat dabei eine starke, traditionsreiche Position zu verteidigen und hat alles Interesse daran, an der Dynamik der Entwicklungsprozesse und Veränderungen der ostasiatischen Entwicklungs-lländer teilzuhaben. 


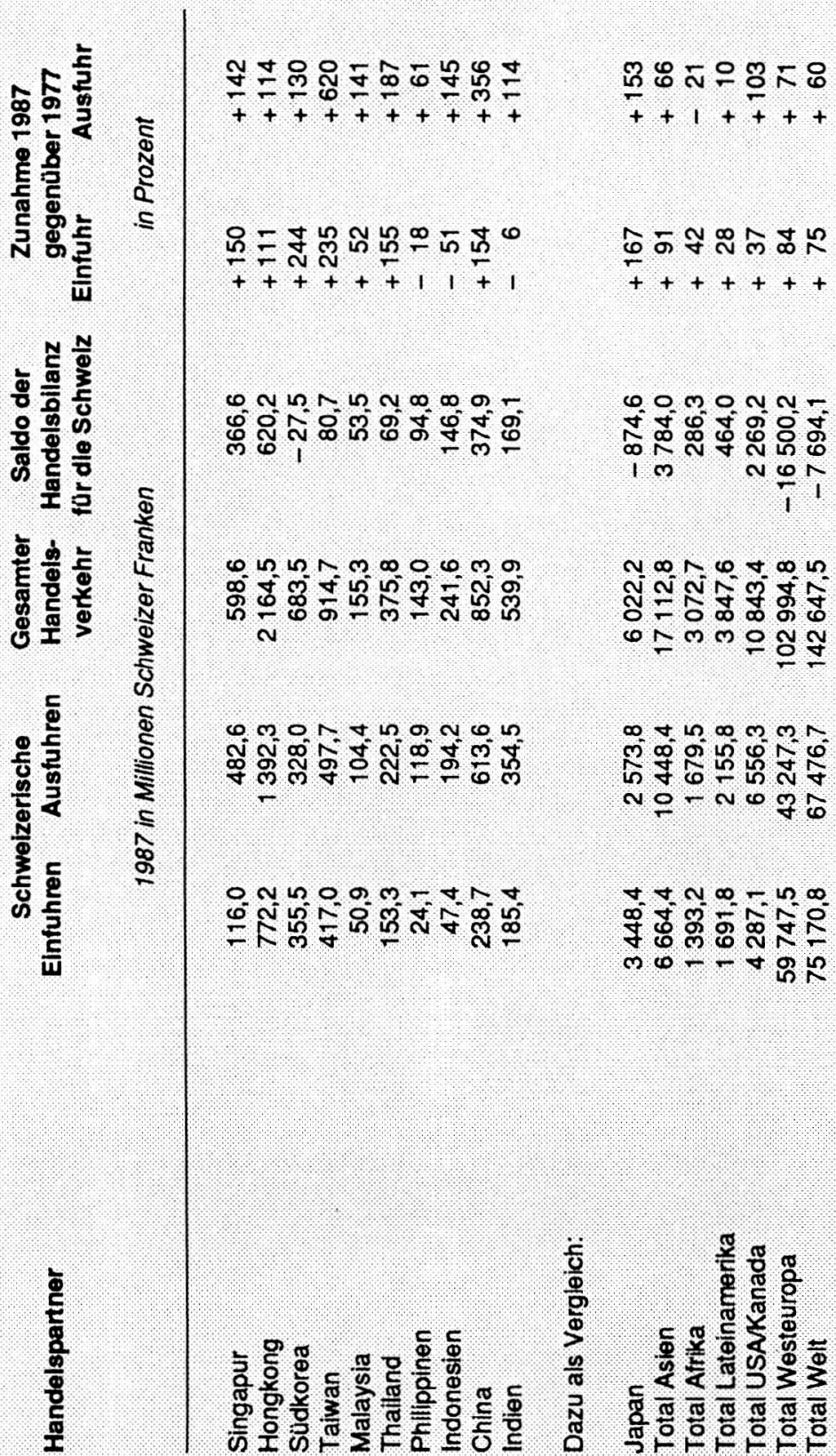




\section{Anmerkungen}

1. vgl. dazu auch Saburo Okita: "The Outlook for Pacific Cooperation and the role of Japan", Japan Review of International Affairs, Spring/Summer 1987

2. Die Angaben über Indien und Bangladesh sind zum Vergleich in gewissen Tabellen miteingeschlossen. Die sozialistischen Länder Südostasiens werden wegen des Mangels an Daten und aufgrund ihres Stellenwerts in den schweizerischen Handelsbeziehungen hier nicht berücksichtigt.

3. The World Bank: World Development Report, 1987

4. Alle statistischen Angaben aus: The World Bank, Development Report, 1987

5. Der Monat, Schweiz. Bankverein, Juli-August 1988, Seite $6 \mathrm{ff}$.

6. China befindet sich derzeit in Beitrittsverhandlungen, ist also in dieser Auflistung noch nicht berücksichtigt.

7. vgl. dazu Rudolf Ramsauer: Swiss Direct Investment and the Association of Southeast Asian Nations, Diss. Institut universitaire de hautes études internationales, Genf 1983

8. vgl. dazu Nicolas Imboden: "Mischfinanzierungen: Entwicklungshilfe oder Exportförderung?", Die Volkswirtschaft 7/88, Bern, S. 14 ff.

9. Bela Balassa: "The importance of trade for developing countries", Banca Nazionale del Lavoro Quarterly Review, January 1988, Seiten 437-469

10. vgl. "Developments in the Trading System (Oct. 1987-April 1988)", Bericht des GATT-Sekretariates, Appendix V

11. "The next Japans", The Economist, 30 July 1988

12. Pat Choate \& Juyne Linger: "Tailored Trade: Dealing with the World as It is", Harvard Business Review, Jan.-Feb. 1988 